\title{
Developing a new methodology to characterize in vivo the passive mechanical behavior of abdominal wall on an animal model.
}

\author{
R. Simón-Allué ${ }^{\mathrm{a}, *}$, J. M. M. Montiel ${ }^{\mathrm{a}}$, J.M. Bellón ${ }^{\mathrm{b}, \mathrm{c}}$, B. Calvo ${ }^{\mathrm{a}, \mathrm{c}}$ \\ a Aragón Institute of Engineering Research (I3A), Universidad de Zaragoza, Spain. \\ ${ }^{\mathrm{b}}$ Department of Surgery, Medical and Social Sciences, Faculty of Medicine and Health Sciences, \\ Universidad de Alcalá, Spain. \\ ${ }^{\mathrm{c}}$ Centro de Investigación Biomédica en Red en Bioingeniería, Biomateriales y Nanomedicina
} (CIBER-BBN), Spain.

\begin{abstract}
The most common surgical repair of abdominal wall hernia goes through implanting a mesh that substitutes the abdominal muscle/fascia while it is healing. To reduce the risk of relapse or possible complications, this mesh needs to mimic the mechanical behavior of the muscle/fascia, which nowadays is not fully determined. The aim of this work is to develop a methodology to characterize in vivo the passive mechanical behavior of the abdominal wall. For that, New Zealand rabbits were subjected to pneumoperitoneum tests, taking the inner pressure from $0 \mathrm{mmHg}$ to $12 \mathrm{mmHg}$, values similar to those used in human laparoscopies. Animals treated were divided into two groups: healthy and herniated animals with a surgical mesh (polypropylene Surgipro ${ }^{T M}$ Covidien) previously implanted. All experiments were recorded by a stereo rig composed by two synchronized cameras. During the postprocessing of the images, several points over the abdominal surface were tracked and their coordinates extracted for different levels of internal pressure. Starting from that, a three dimensional model of the abdominal wall was reconstructed. Pressure-displacement curves, radii of curvature and strain fields were also analysed. During the experiments, animals tissue mostly deformed during the first levels of pressure, showing the noticeable hyperelastic passive behavior of abdominal muscles. Comparison between healthy and herniated specimen displayed a strong stiffening for herniated animals in the zone where the high density mesh was situated. Cameras were able to discern this change, so this method can be used to measure the possible effect of other meshes.
\end{abstract}

Key words: Abdominal wall; Mechanical properties; In-vivo studies; Photogrammetry;

* Corresponding author. Mechanical Department. c/ María de Luna s/n 50018. Zaragoza. Spain. Email address: rsimon@unizar.es, Tel.: +34 876555187 


\section{Introduction}

Abdominal wall hernia is a common health problem which affects more than 20 million people per year around the world (Dabbas et al., 2011; Kingsnorth and LeBlanc, 2003). An hernia consists on a protusion of inner organs through the structure that usually contains it. In the case of abdominal wall, a weakness in the wall may lead to an hernia due to the loss of equilibrium between the internal pressure and the active and passive forces of the abdominal cavity (Dumanian, 2007). Its treatment for severe cases lies in implanting a surgical mesh which substitutes the abdominal muscle/fascia throughout the time it is healing. In order to avoid the risk of tear or the excessive laxity of the tissue after the surgery, this mesh has to mimic the mechanical behavior of the abdominal wall in its physiological range.

Although this type of surgery has been widely applied, cases of hernia relapses and the appearance of some common problems such as fibrosis (Klinge et al., 2002; Klosterhalfen et al., 2002; Cobb et al., 2005) or mesh-tissue adhesions are not unusual. The presence of pain after the mesh insertion (Paajanen and Hemunen, 2004) or even the tear of the mesh are also possible side effects of the intervention. The way to reduce them involves acquiring a better understanding of the abdominal wall and its mechanical behavior when muscle, fascia, tendons and skin are working together.

Many previous studies have been focused on mechanical characterization of isolated tissues that compound the abdominal cavity, i.e. abdominal fascia, individual abdominal muscles or skin. Most of them have been performed on animal models, using pigs (Lyons et al., 2014; Cooney et al., 2015), dogs (Greca et al., 2001; Hwang et al., 2005) or rabbits (Hernández et al., 2011; Peeters et al., 2013), and a few others on humans (Kirilova et al., 2011; Förstemann et al., 2011; Martins et al., 2012; Ben Abdelounis et al., 2013). Nevertheless, when isolating one determined tissue from the whole, it may change the mechanical response of this tissue due to a loss of mechanical resistance. Therefore, new testing methods that allow the abdominal cavity to be characterized as a whole, i.e. when all components are working together, are needed to better determine its mechanical behavior. Particular highlights in this regard are Podwojewski et al. (2014) and Tran et al. (2014), who carried out ex vivo mechanical tests on human specimens considering the contribution of the different layers, and Song et al. (2006) and Szymczak et al. (2012), who performed in vivo tests measuring on a live human abdomen while this was subjected to different stresses. All these works, however, have some differences or limitations. Both Podwojewski (Podwojewski et al., 2014) and Tran (Tran et al., 2014) works were based on ex vivo tests instead of in vivo, and defrosted human specimens were used without considering frozen effects on the mechanical response (Clavert et al., 2001; Giannini et al., 2008). Szymczak (Szymczak et al., 2012) studied strains in some points of the specimen but stress was originated from muscle contraction, so active response of the muscle was studied there. At last, Song presented a working scheme similar to the one followed here, but it was limited to healthy specimens so the hernia effects could not be analyzed.

The goal of this study is to develop an in vivo methodology that allows to evaluate the contribution of the abdominal wall tissues on the mechanical response of the wall. To achieve this 
aim, in vivo mechanical tests were performed in conjunction with imaging methods so that strain information could be obtained from the tissue in a non-invasive way. In vivo experiments consisted on the insufflation of carbon dioxide (pneumoperitoneum) into the abdominal cavity by laparoscopy, a technique largely used in hernia surgery, while observing the abdomen deformation with a stereo pair of digital cameras. The protocol implemented here was carried out on an animal model, specifically on New Zealand rabbits, species frequently used in the study of surgical hernia repair (Bellón et al., 1996; LeBlanc et al., 2002; Hernández et al., 2011; Simón-Allué et al., 2014).

\section{Material and Methods}

\subsection{Specimens}

For the fulfillment of this study, four New Zealand rabbits were subjected to several pneumoperitoneum tests. Animals were obtained from the Animal Experimentation Service of the Research Support Services of the University of Zaragoza, with an average weight ranged from 2 to $2.3 \mathrm{~kg}$. They were housed separately, and were watered and fed a standard chow diet ad libitum (Finished feed n. 511; Food Corporation Guissona S.A., Lleida, Spain).

Animals were healthy and free of clinically observable diseases. Previous to the procedure, they were kept under stable conditions of light and temperature following the recommendations given by the Guide for the Care and Use of Laboratory Animals. All procedures were carried out under Project Licence 01/11 approved by the in-house Ethics Committee for Animal Experiments of the University of Zaragoza.

Experiments were divided into two groups. The first group was composed by two healthy specimens while the second group, conversely, was consisted of two herniated animals with a surgical mesh previously implanted. Animals were intramuscularly anaesthetized with a mixture of medetomidine $\left(0,14 \mathrm{mg} / \mathrm{kg}\right.$, Medeson $^{\circledR}$; Uranovet, Barcelona, Spain), ketamine $(20 \mathrm{mg} / \mathrm{kg}$, Imalgene $1000^{\circledR}$; Merial Laboratorios S.A., Barcelona, Spain) and butorphanol $(0,3 \mathrm{mg} / \mathrm{kg}$, Torbugesic ${ }^{\circledR}$; Fort Dodge Veterinaria S.A., Girona, Spain).

Hernia disease consisted of a total defect on the abdominal wall encompassing all abdominal layers (muscles, fascias and parietal peritoneum) and being replaced and repaired with a high density, non-absorbable surgical mesh made by polypropylene (Surgipro ${ }^{T M}$, Covidien) with the size of $5 \times 7 \mathrm{~cm}$. Tissue - mesh attachment was conducted by a continuous 4-0 polypropylene suture interrupted in the implant angles. This mesh was inserted one week before the pneumoperitoneum tests and its location on the animal is indicated in Fig. 1. Skin was closed with a 2-0 polypropylene suture. Once the wound was closed, the visible scar was a 100-mm incision along the linea alba direction (see Fig. 2. b).

Before the test, specimens were cropped from front to rear legs in order to clear the visibility of 
the external abdominal cavity. Animals were placed over the surgical table in supine position, with the legs fixed to the table and the abdominal surface completely exposed. This surface was spotted with black dots which were used as fiducial markers to be tracked during the experiment. Fiducial markers had an average diameter of $8 \mathrm{~mm}$ and covered an almost diamond-shaped useful area of $100 \mathrm{~cm}^{2}$. Coded targets were also added to the surgical table to define a reference for the 3D location estimates. Adding these coded markers ensures that 3D measurements correspond to the very same abdominal points along the whole range of pressures. Final setup is shown in Fig. 3.

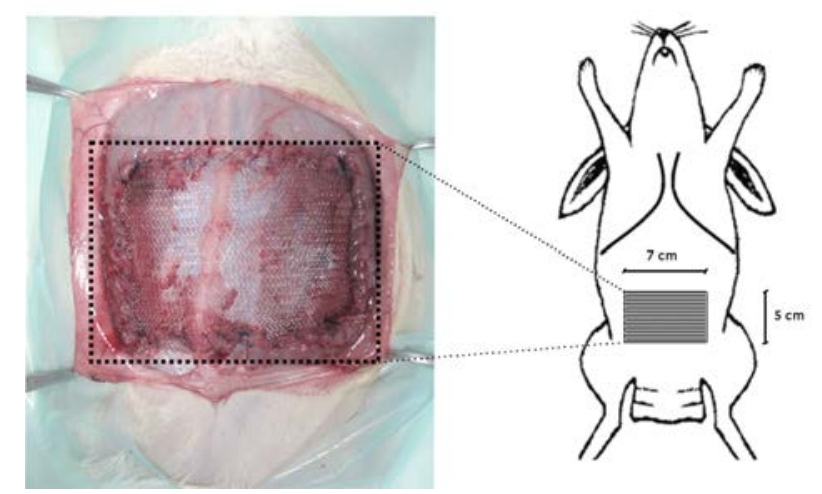

Fig. 1. Defect on the abdominal wall repaired with a synthetic mesh and location on the specimen.

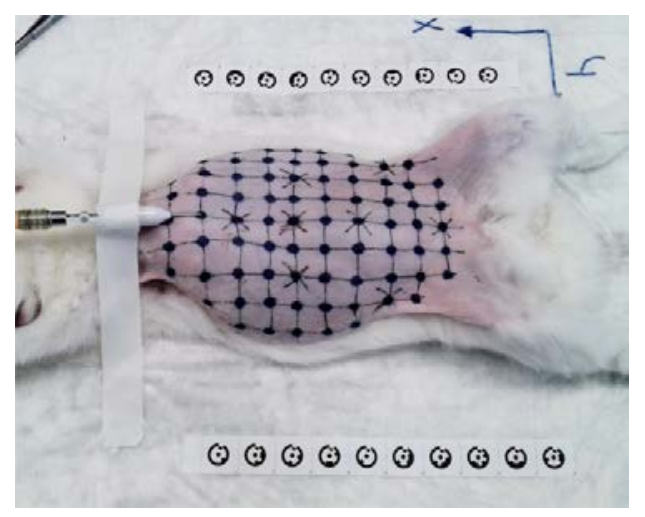

(a) Healthy animal.

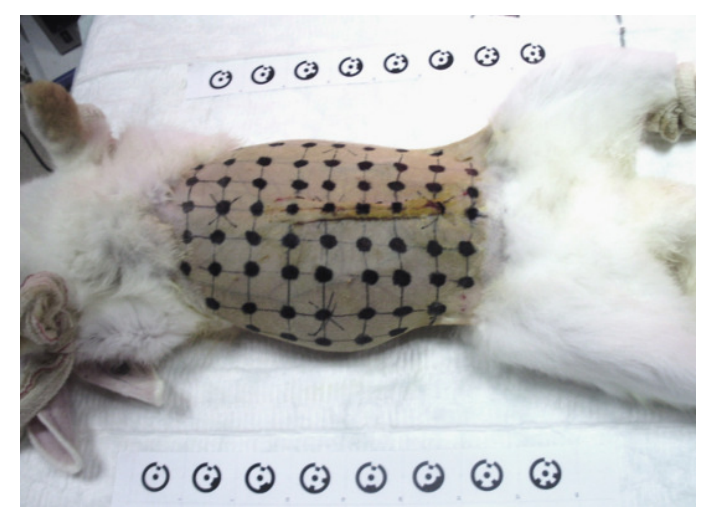

(b) Herniated animal.

Fig. 2. Specimen preparation for the recorded pneumoperitoneum test. A tattooed grid was realized on the abdominal wall of the rabbit.

\subsection{Cameras}

The procedure was recorded by a synchronized stereo rig, composed by two Prosilica GT1290 cameras with a Sony ICX445 EXview HAD CCD sensor. Image sequences were black and white having a 1280x960 resolution at a 5 fps frame rate stored in raw format to avoid compression losses.

The stereo rig was situated over the specimen so that both cameras were able to cover all the 
abdominal surface exposed. This was specially significant since 3D location estimates can be provided only for those points imaged in both cameras. Another determining factor for the goodness of the measurements was the parallax, which makes reference to the angle formed between cameras position in relation to the observed marker. In order to minimise the measurement error, this angle has to be as closer as possible to $90^{\circ}$, which is practically the value achieved in the experimental setup for all the markers (see Fig. 3). A prior camera calibration was needed to establish the intrinsic parameters of the camera and its internal geometry. A planar pattern method similar to Bouguet (2011) was applied. For this geometry and camera resolution an accuracy better than $1 \mathrm{~mm}$ can be assured.

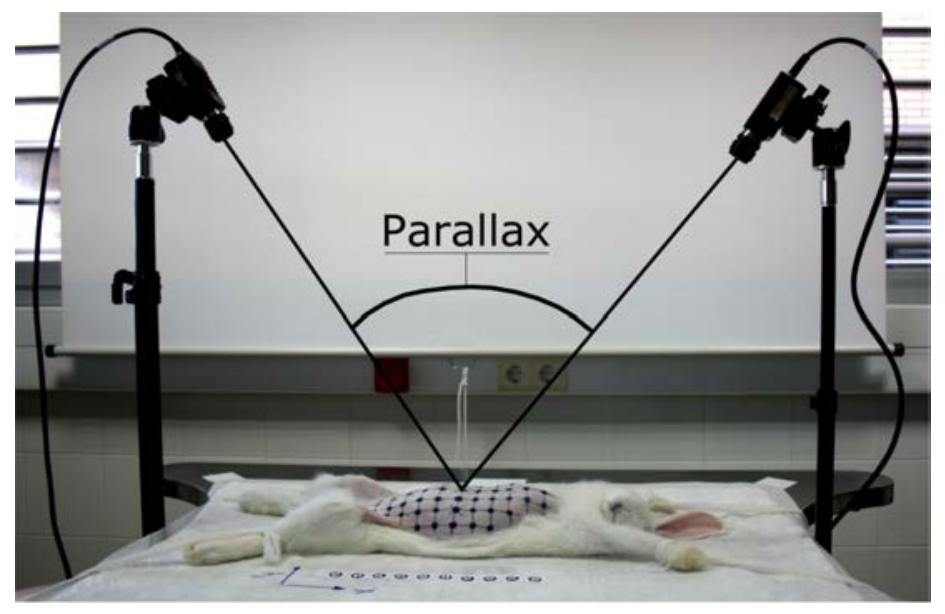

Fig. 3. Experimental setup: Animal and cameras arrangement during the experiment.

\subsection{Experiment}

Once the animal and cameras were prepared, a Veress needle was inserted in the upper abdomen and fixed to a external reference. This needle was used for introducing carbon dioxide inside the cavity and was selected instead of the trocar for being considered less invasive during the experiment. In addition to this, a Standard Karl Storz endoscope was utilized for the gas supply. The experiment consisted of conducting a pneumoperitoneum in the abdominal cavity of the animal with carbon dioxide in a controlled manner, so that the deformed shape corresponding to determinate levels of pressure could be identified and extrapolated from the video. During the experiment, animals were kept alive but completely anesthetized. They were not intubated or ventilated, in order to not to modify the pressure level measured. The heart was beating with small rate, but blood pressure was considered irrelevant in terms of mechanical response of the wall.

Three tests were performed on the same specimen, returning to the zero pressure state after each test. To ensure that, the pressure value inside the cavity was checked using a Fiber Optic Pressure sensor $\left(\right.$ Opsens $\left.{ }^{\circledR}\right)$. The sensor was introduced in the opposite side of the cavity, at initial and final time of the procedure. The first test of the same specimen was excluded from the study due to the preconditioning, so two tests per specimen were selected for the analysis of 
the results. A total of four pneumoperitoneum tests were analysed for each group, healthy and herniated rabbits. During the experiment, the endoscope insufflated carbon dioxide increasing the inner pressure of the abdominal cavity from 0 to $12 \mathrm{mmHg}$, according to the human values commonly used in laparoscopy. In order to obtain data along the experiment the addition was carried out in 12 steps, one per $\mathrm{mmHg}$, so the cameras could get images from given levels of pressure. In the cases conducted on herniated animals only 6 steps were able to be performed, obtaining pressure and frames data each $2 \mathrm{mmHg}$.

\subsection{Data postprocessing}

The digital photogrammetry PhotoModeler software (Pho, 2013) was used for processing the images. From the recorded video, a stereo pair of frames was selected for each level of pressure and these frames were processed to estimate the 3D coordinates of the fiducial markers over the abdominal surface and over the surgical table. Hence 3D locations of the points were obtained along the experiment, i.e. for different levels of pressure.

A total of 43 points from the abdominal surface were tracked for the analysis of the results. The three-dimensional coordinates were used to evaluate the displacements of those points, as well as to develop a 3D model of the cavity based on the reconstruction of the geometry for different pressures. From this model, principal radii of the surface were estimated using the 3D modelling software Rhinoceros (Rhi, 2014). Longitudinal and transversal directions were also distinguished during the post processing and sections in both directions were displayed to analyse the evolution with the pressure increase. Pressure-displacement curves of five main points of the surface were also constructed.

\section{Results}

A healthy specimen is shown at the initial (see Fig. 4(a)) and final (see Fig. 4(b)) moment of the experiment. Tracking of dots enabled to follow the evolution of abdominal displacement with the level of pressure. Inflation tests showed a maximum displacement of $42.3 \mathrm{~mm}$ in healthy animals and significantly lower, $28.65 \mathrm{~mm}$, in the case of animals repaired with the Surgipro ${ }^{T M}$ high density mesh for $P=12 \mathrm{mmHg}$. Both values were measured at the black dot corresponding to the center of the abdominal surface. The main component of the displacement was performed in the vertical direction, being the maximum value up to $2.5 \mathrm{~mm}$ in the $X$ (longitudinal) direction for points on the lower abdomen, and $8 \mathrm{~mm}$ in the $Y$ (transverse) direction for points on the side of the abdomen. A greater displacement was observed in points of the lower abdomen, towards the rear legs, rather than points of upper abdomen, closer to the head and the front legs. This difference probably lies in the limiting effect of the rib cage that prevents the upper abdomen from a severe deformation.

All the experiments run in a similar way showing the maximum gradient of deformation during 


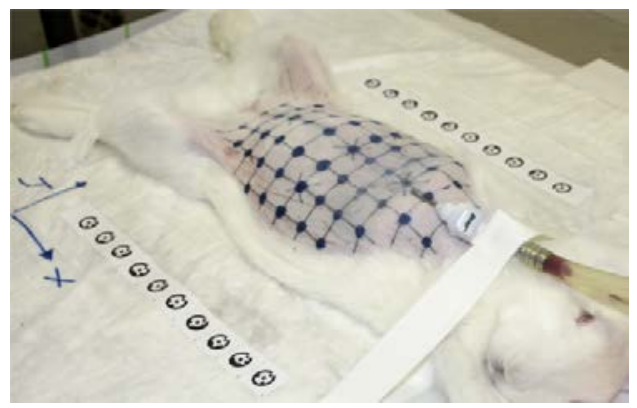

(a) $P=0 \mathrm{mmHg}$

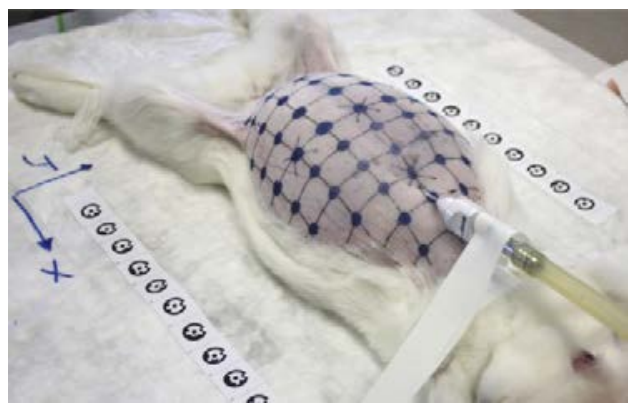

(b) $P=12 \mathrm{mmHg}$

Fig. 4. Healthy animal in the initial (a) and final (b) instant of the inflation test.

the first four or five mmHg of pressure increase. However, it was noted that the first test performed on the same specimen was fully different from the following. In this first test gas was inserted without causing an increase of pressure in the beginning, and the amount of gas needed to observe visible changes on the abdominal cavity was much greater than in the following tests. From the second experiment onwards, tests showed a similar and reproducible behavior. For that reason, the first test on every specimen was considered preconditioning and was excluded from the study.

\subsection{Pressure - Displacement curves}

With the purpose of analysing the mechanical response of the abdominal wall, five main dots were studied and compared in depth, corresponding to the central, sides, upper and lower zones of the abdominal surface (see Fig. 5(a)). Pressure - Displacement curves for these dots with mean and standard deviation of the four tests performed are shown in Fig. 5(b) and Fig. 6(b)for healthy and herniated animals respectively. Horizontal axis refers to the inner pressure reached in the abdominal cavity and vertical axis plots the displacement of each point, calculated from the coordinates transformation in the three principal axis. Greater values of displacement for higher levels of pressure were observed in healthy animals rather than animals with a surgical mesh inserted. Specifically, a reduction of almost $30 \%$ was observed in points of maximum displacement between healthy and herniated cases (e.g. Point 2), while the value remained similar in points closer to the upper legs (e.g. Point 1).

In Fig. 6(a), the exact place where the surgical mesh was inserted is appreciated. According to the pressure - displacement curves the presence of the mesh leaded to an increase of stiffness in the lower zone of the abdomen, where the mesh was inserted. This implies a smaller deformation of the muscle and therefore, a lower dispersion on the displacement values for different points of the surface. As a result, curves from Fig. 6(b) remained closer together than in the case of healthy animal, Fig. 5(b).

Fig. 7 compares the displacement obtained for the central point of the surface (Point 2) for 


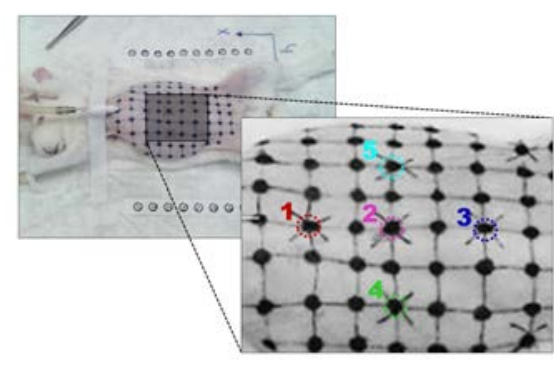

(a) Reference points of the surface.

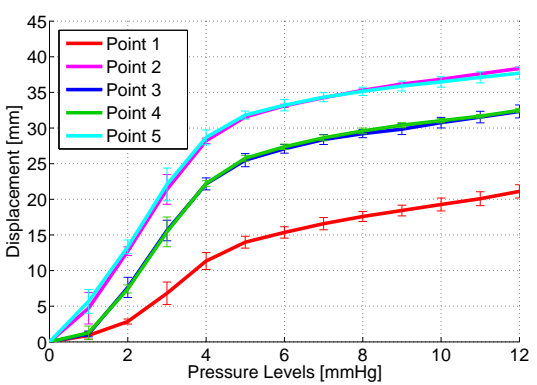

(b) Pressure - Displacement curves. Mean and standard deviation of healthy specimens.

Fig. 5. Pressure - Displacement curves for main points of the healthy abdominal surface.

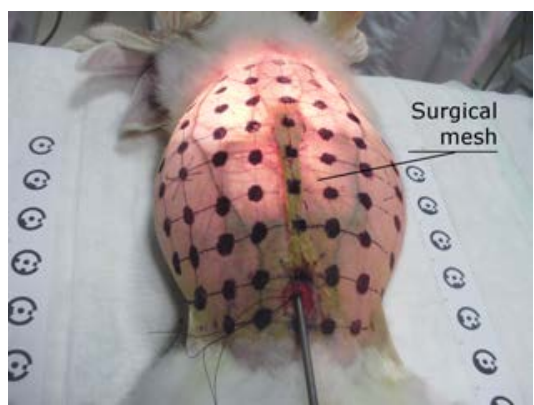

(a) Surgipro ${ }^{\circledR}$ mesh location inside the herniated animal.

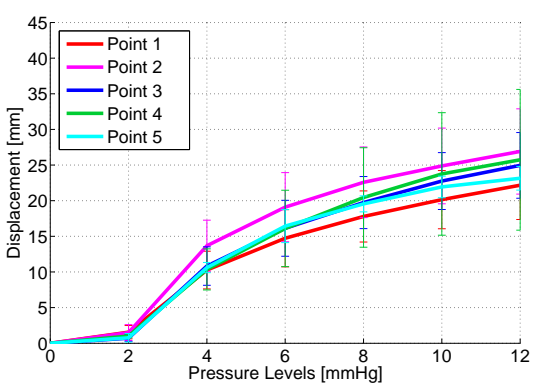

(b) Pressure - Displacement curves. Mean and standard deviation of herniated specimens.

Fig. 6. Pressure - Displacement curves for main points of the herniated abdominal surface repaired with Surgipro ${ }^{\circledR}$ mesh.

both groups of animals, healthy and herniated repaired with Surgipro ${ }^{T M}$ mesh. For that, means and standard deviations are displayed.

\subsection{D Reconstructions}

Based on the three-dimensional coordinates obtained from video frames along the experiment, a reconstruction of geometry was carried out for some levels of pressure, $\mathrm{P}=0,3,6,9$ and $12 \mathrm{mmHg}$ (see Fig 8(a)). For these reconstructions, the coordinates of the dots were respected, and a smooth surface passing through the points was generated with the help of the modelling software Rhinoceros. The rest of the model was generated manually taking into account geometrical considerations of the animal and the animal-table set in the experimental set-up.

Sections in both principal directions, longitudinal and transverse plane, were also performed 


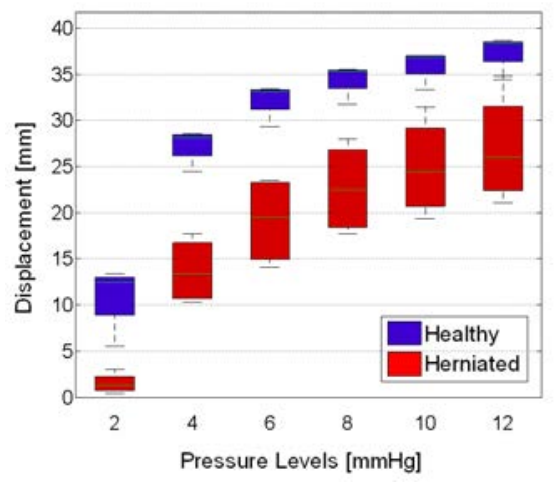

Fig. 7. Displacements of the central point of the abdominal surface. Comparison between results of healthy and herniated specimens.

on the reconstructed model (see Fig. 8(b)). In Fig. 8(c) the progress of these sections with the pressure is shown. According to these outlines, the cavity deforms the most during the first levels of pressure, reaching the $80-90 \%$ of the total strain at $P=6 \mathrm{mmHg}$.
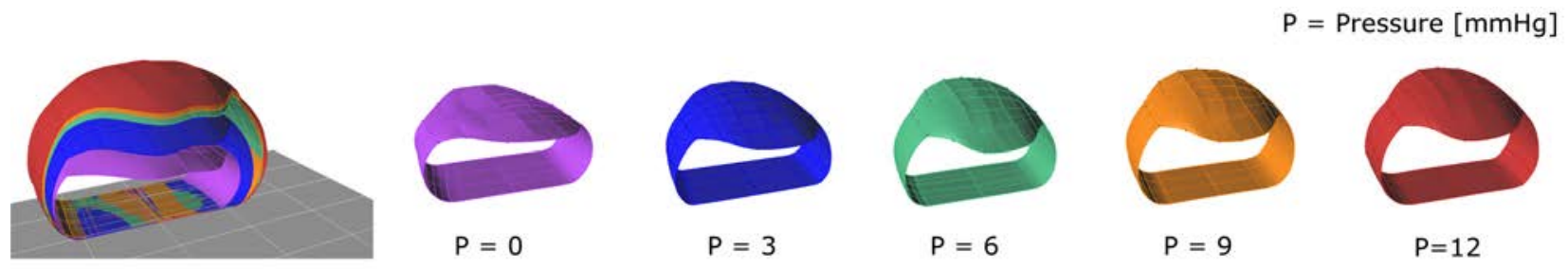

(a) $3 \mathrm{D}$ reconstructions according to the pressure level.

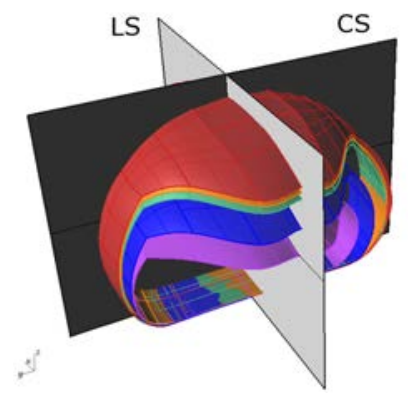

(b) Geometry sections.
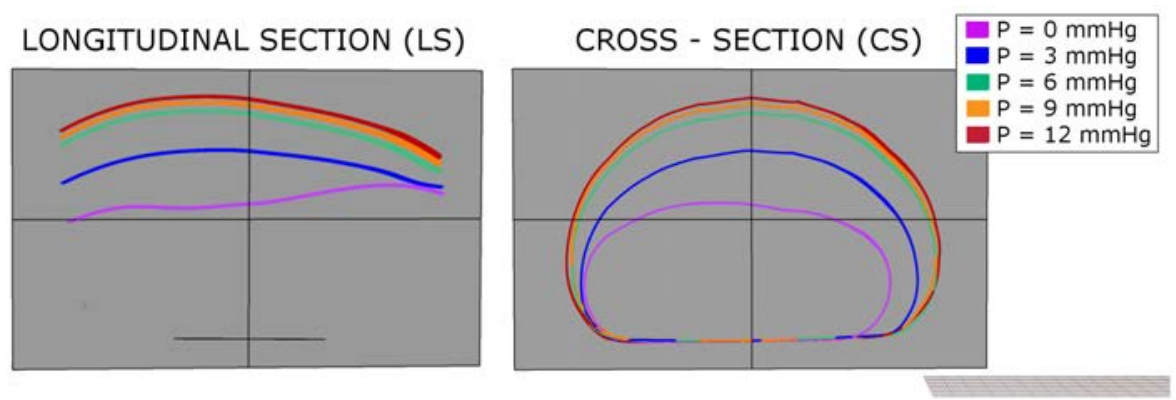

(c) Progress of the Longitudinal and Cross-Section with the pressure. $\mathrm{LS}=$ Longitudinal Section. CS=Cross-Section

Fig. 8. 3D reconstruction of the cavity for different inflation pressures.

Principal radii of curvature were also estimated based on the three-dimensional model reconstructed for several levels of pressure (see Fig. 9(a)). The evolution of the radii throughout the carbon dioxide insufflation, i.e. pressure increase, is observed in Fig. 9(b). According to this, the curvature change in the longitudinal direction was bigger than in transverse direction. 


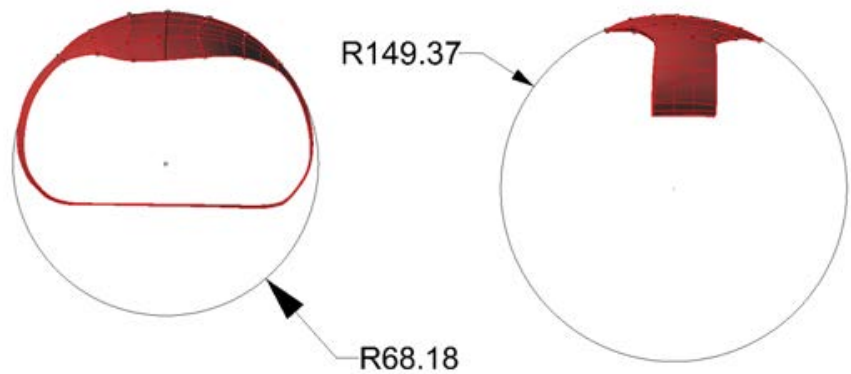

(a) Radius measurements (in $\mathrm{mm}$ ) taken from the $3 \mathrm{D}$ model for $\mathrm{P}=12 \mathrm{mmHg}$.

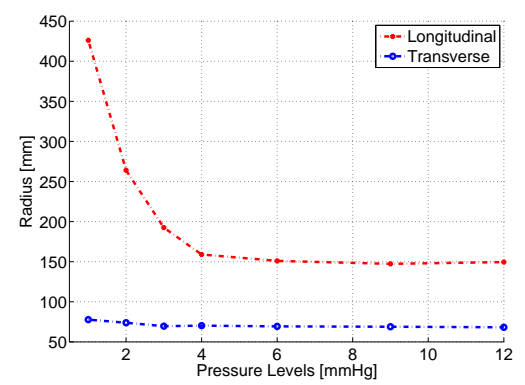

(b) Longitudinal and transverse radii vs. level of pressure.

Fig. 9. Radii of curvature changes during carbon dioxide insufflation.

\subsection{Stretching data}

Stretching values in the principal directions were also studied for $12 \mathrm{mmHg}$ of pressure. Fig. 10 shows the segments along the sections where the elongation was analysed and values are specified in Table 1.

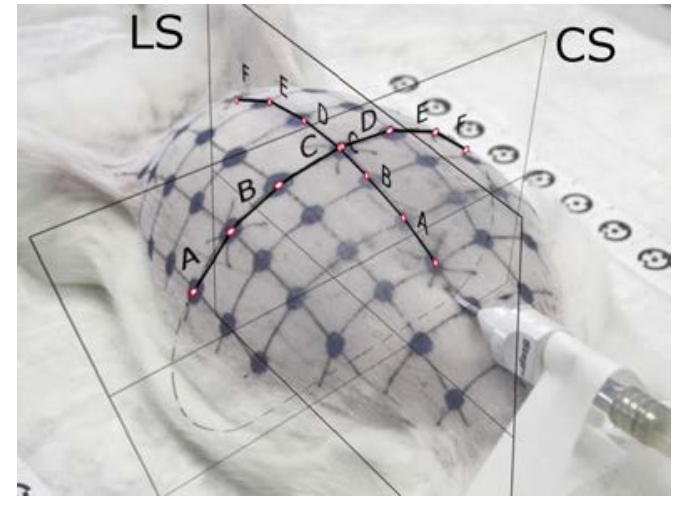

(a) Segments along the principal sections.

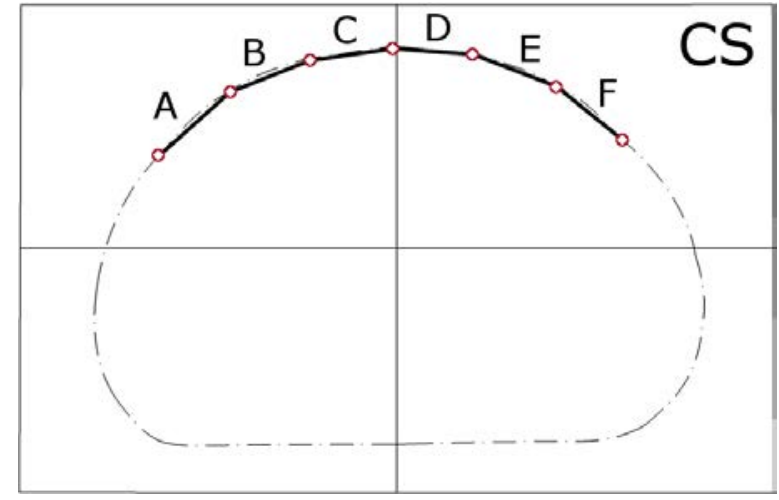

(b) Cross-Section detail.

Fig. 10. Sections segments where the stretching was analysed.

Lagrangian strain maps were also calculated from displacements of fiducial dots. To do that, a rough mesh of quadrilateral elements was constructed over the abdominal surface where fiducial points worked as nodes of the mesh. This way, displacement of each point in the area was calculated from the nodal values, $\mathbf{u}^{\text {nod }}$, and the approximating functions of a bilinear quadrilateral, $\mathbf{N}(x, y)$, as $\mathbf{u}(x, y)=\mathbf{N}(x, y) \cdot \mathbf{u}^{\text {nod }}$. Lagrangian strain tensor $\mathbf{E}$ was calculated at Gaussian points in each quadrilateral element as 
Table 1

\begin{tabular}{|c|c|c|c|c|c|c|}
\hline Transverse & A & B & C & D & E & F \\
\hline Healthy & 22.41 & 20.33 & 3.23 & 3.01 & 15.42 & 19.85 \\
\hline Herniated & 15.12 & 14.19 & 3.44 & 6.77 & 9.46 & 15.15 \\
\hline \hline Longitudinal & $\mathbf{A}$ & $\mathbf{B}$ & $\mathbf{C}$ & $\mathbf{D}$ & $\mathbf{E}$ & $\mathbf{F}$ \\
\hline Healthy & 4.11 & 2.00 & 1.37 & 3.13 & 5.41 & 8.74 \\
\hline Herniated & 4.03 & 6.25 & 2.89 & 1.54 & 2.97 & 2.39 \\
\hline
\end{tabular}

Mean stretching for $12 \mathrm{mmHg}$ in the transverse and longitudinal direction (\%) according to the segments delimited in Fig. 10.

$$
\mathbf{E}(x, y)=\frac{1}{2}\left[\mathbf{F}^{T}(x, y) \cdot \mathbf{F}(x, y)-\mathbf{I}\right]
$$

where $\mathbf{F}$ is the deformation gradient tensor obtained as $\mathbf{F}=\frac{\partial \mathbf{x}}{\partial \mathbf{x}}=\mathbf{I}+\frac{\partial \mathbf{N}}{\partial \mathbf{x}} \mathbf{u}^{\text {nod }}$, with $\mathbf{N}$ the shape function for the bilinear quadrilateral, $\mathbf{u}^{\text {nod }}$ the nodal displacements, $\mathbf{X}$ the reference configuration and $\mathbf{I}$ the identity matrix.

A general strain map is displayed in Fig.11(a) and strain in both principal directions, corresponding to the components $E_{11}$ and $E_{22}$ of the tensor $\mathbf{E}$, is plotted in Fig. 11 (b) and (c). Transversal components $E_{12}$ and $E_{21}$ of the tensor were close to zero which supports the hypothesis that the wall is working as a membrane, i.e., only in principal directions.

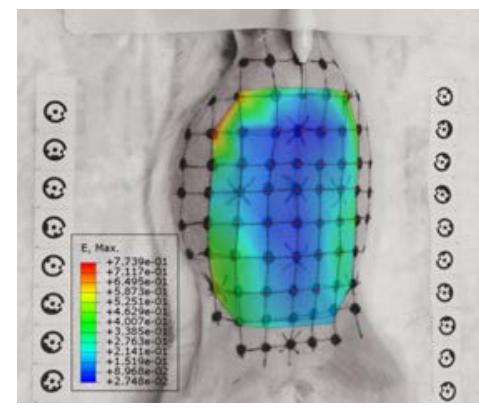

(a) Strain map along the abdominal surface.

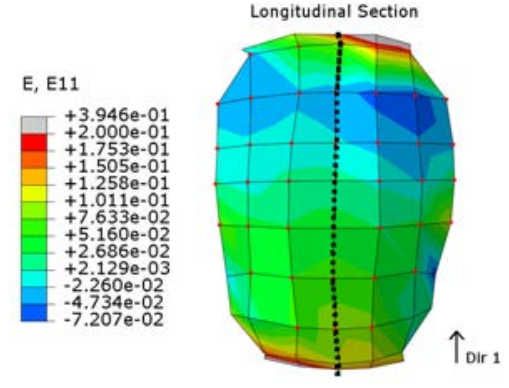

(b) Longitudinal direction.

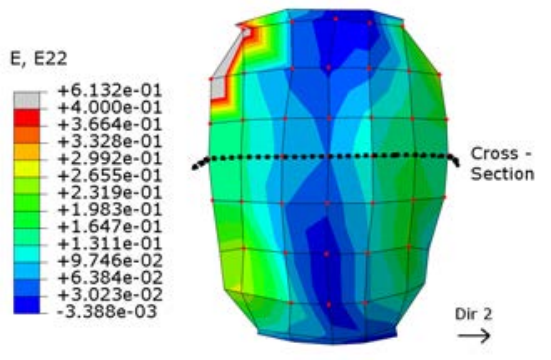

(c) Transversal direction.

Fig. 11. Lagrangian strain maps on the abdominal surface of a healthy animal based on the fiducial points tracked.

Stress values in the principal directions were calculated using Laplace's Law and approximating the animal abdominal wall to an ellipsoidal shape, similar to a truncated rugby ball. Applying the same criteria in both principal directions, longitudinal and transversal stresses were estimated for each level of pressure and represented with regard to the stretching of the different segments along the sections (see Fig. 12). 


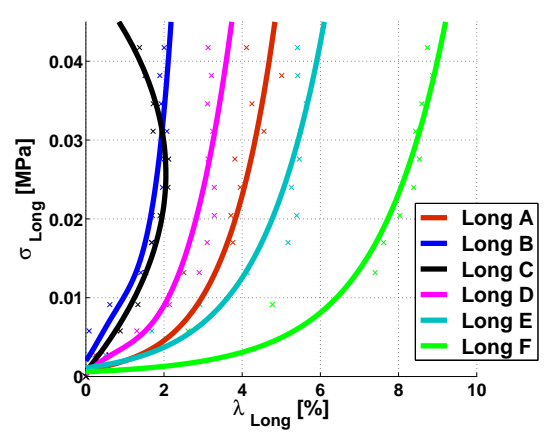

(a) Longitudinal direction.

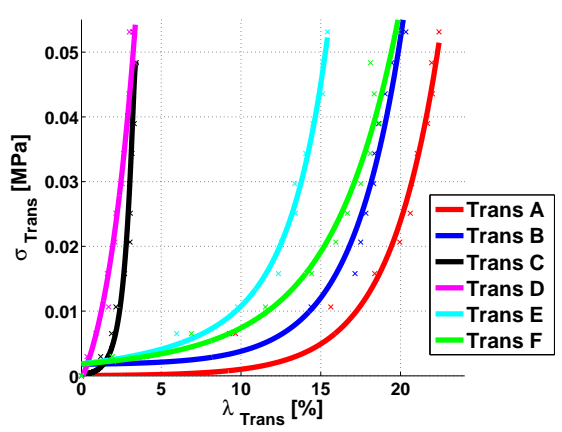

(b) Transversal direction.

Fig. 12. Cauchy Stress-Strain relationship for both principal directions.

\section{Discussion \& Conclusions}

In the present work, in vivo mechanical behavior of abdominal wall was studied on an animal model. For that, several pneumoperitoneum tests were carried out on two groups of rabbits, composed of healthy specimens and herniated ones with a surgical high density mesh previously inserted. All experiments were recorded using a stereo system which enabled, by postprocessing of the images, to extract three-dimensional coordinates of different points highlighted on the abdominal surface. Based on that information, the geometry of the cavity was successfully reconstructed for different levels of pressure. Pressure-displacement curves for reference points, principal radii of curvature and stretching in principal planes were also calculated.

Experiments first showed differences between the initial inflation test performed on each specimen and the following. This may be related to a distribution of the gas inside the cavity, which contributed to squeeze internal organs before deforming the abdominal wall. These differences were also noted by Song et al. (2006), who associated this phenomenon to an unobserved internal reshaping in the form of diaphragm movement.

Pressure - displacement curves confirmed the strong non-linear mechanical behavior when the whole abdominal wall is working. At low pressures, muscle deformed considerably for each level of pressure. At high pressures, the tissue got more rigid and the strain increment for each mmHg of pressure was much smaller (Fig. 5(b) and 6(b)). It was also shown in Fig. 9(b), when the main change of the principal radii happened during the first half of the experiment. This is associated with the fact that the abdominal muscles are considered an extracellular matrix with muscular and collagen fibers inserted, responsible for force generation and resistance of the tissue respectively. For low stresses collagen fibers present a wavy pattern which endows the tissue with a slight resistance, but it becomes more rigid when collagen fibers are oriented and stretched at higher pressures (Hernández-Gascón et al., 2011, 2012).

Differences between stretching of segments in each direction (see Table 1) were explained taking account the rabbit anatomy. The longitudinal section was measured along the linea alba, 
where the junction between fascias and rectus abdominis lies moderately constant and the small differences between stretching of the segments can be explained due to the changes in the stretch response of the linea alba along its length (Cooney et al., 2015). However, crosssection goes through three different composite muscle layers besides the linea alba: rectus abdominis (segments C \& D), External-Internal composite (segments B \& E) and ExternalInternal-Transversal composite (segments A \& F) (Hernández-Gascón et al., 2011). The distinct mechanical response of these three composites could explain the differences of stretching founded between segments.

Attending to the effect of the surgical mesh on the mechanical behavior of the abdominal wall, the study showed a strong stiffening on the part of the surgical mesh which caused a decrease of deformation of the wall during the experiment. This stiffening was visible in Fig 7 , where the herniated line kept below the healthy one in all pressure levels, and also in the stretching values gathered in Table 1. For herniated animals, values obtained in the transverse direction were lower than the healthy ones due to the high density mesh rigidity. Similar results were found for the longitudinal plane, but only in segments D, E and F, where the mesh was situated. It is also noticed that some regions showed a lower tissue stretching in healthy animals rather than the herniated ones. It could be that the presence of the mesh, which stiffens some segments (e.g. longitudinal segments D, E \& F), leads in a pull to the adjacent segment (e.g. segment C) provoking an increase of stretching in this area.

Some authors have previously studied the mechanical response of abdominal tissue when it has been repaired with a surgical mesh. Podwojewski et al. (2013) reported that there were not significant differences in stiffness between intact specimens and repaired with a surgical mesh, but that was a polyester Parietex ${ }^{T M}$ Composite, a synthetic mesh different from the Surgipro ${ }^{\circledR}$ used here. Henández et al. (Hernández-Gascón et al., 2011, 2012) studied the mechanical behavior of the Surgipro ${ }^{\circledR}$ mesh together with other synthetic surgical meshes and both studies ended with the assumption that the Surgipro ${ }^{\circledR}$ mesh leads to suppose an increase in stiffness. The methodology developed in this work was able to measure this stiffening in a complete non invasive way, and could be used with other meshes after surgery to determine if they are affecting the rigidity of the abdomen. This factor is important since it is directly related to the patient comfort.

According to the strain field measured over the abdominal surface, the longitudinal direction (measured over the linea alba) seemed to be stiffer than the transversal one (measured halfway on the abdominal surface). This fact may appear to be opposed to previous studies in abdominal muscle, but actually there is a great dispersion about this issue in literature and some differences should be explained. Hwang et al. (2005) agreed with this assumption in his work about canine abdominal muscles, which has a special relevance considering that is a quadruped animal case. Also Kirilova et al. (2011) and Martins et al. (2012) had the same results studying mechanical behavior of fascias and rectus sheaths respectively, but working on human samples and when each element was working alone.

On the other side, some authors (Förstemann et al., 2011; Tran et al., 2014; Podwojewski et al., 2014) found a higher compliance in the longitudinal than in the transverse directions 
when performing in vitro and ex vivo tests of the whole abdominal muscle samples of human. Similar results were found in Song et al. (2006) with the only in vivo experiment, according to author's knowledge, performed on abdominal human tissue. This disagreement could be explained considering that different types of species are being compared here, biped and quadruped animals, so that muscle elongations between them could differ due to the posture. Also Hernández-Gascón et al. (2011), who performed in vitro tests on different composite layers of abdominal rabbit muscle, proved a stiffer behavior in the transverse direction for all composites. Nevertheless, those tests were carried out until failure, which means a higher level of stress than the obtained in this study as physiological range $(\sigma \simeq 0.06 \mathrm{MPa})$. If strain values for physiological stresses are analysed in that study longitudinal reveals to be stiffer than the transversal direction, which is consistent with the findings observed in this work.

This experimental study is not exempt from some limitations. First of all, the number of specimens used for inflation tests should be increased in order to obtain a certain pattern to compare healthy and herniated animals. Different types of synthetic meshes could be tested, so the convenience of distinct materials, pore sizes or thread arrangement would be analysed. Regarding the measurement process, the number of cameras could be increased. This way the measured area along the abdominal wall would grow and the strain of the back muscles of the wall could also be studied. Besides, the post processing of the images needs to be automatized to speed up the obtainment of the 3D model. Finally, this measurement method should be tried on human tissue, since the main objective of this methodology is to be applied in hernia diseases on human beings.

Despite these limitations, this work presents a complete non-invasive method to measure passive mechanical behavior of abdominal muscle. Once the process was automatized, this method could be easily included in a surgical room during the opening of a laparoscopy, and report during the same procedure mechanical information of the specific patient. This information could be very useful for surgeons to choose the surgical mesh that better reproduces the mechanical behavior of the abdominal muscle of the patient.

\section{Conflict of interest statement}

No financial support from other organizations unless the cited in the acknowledgements section, nor personal relationships, nor people, nor organisms biased the development of this research work.

\section{Acknowledgments}

The authors gratefully acknowledge research support from the Spanish Ministry of Economy and Competitiveness through research projects DPI2011-27939-C02-01/DPI2011-27939-C02-02 
and DPI2012-32168. We also thank the Spanish Ministry of Economy and Competitiveness for the financial support to R. Simón-Allué through the grant BES-2012-053422.

\section{References}

(2013). PhotoModeler Scanner. Eos Systems Inc., Vancouver, Canada.

(2014). Rhinoceros 3D. McNeel Europe, Barcelona, Spain.

Bellón, J. M., Buján, J., L. Contreras, L. Carrera-San Martín, A., and Jurado, F. (1996). Comparison of a new type of polytetrafluoroethylene patch (mycro mesh) and polypropylene prosthesis (marlex) for repair of abdominal wall defects. J Am Coll Surg, 183:11 - 18.

Ben Abdelounis, H., Nicolle, S., Otténio, M., Beillas, P., and Mitton, D. (2013). Effect of two loading rates on the elasticity of the human anterior rectus sheath. Journal of the Mechanical Behavior of Biomedical Materials, 20:1 - 5 .

Bouguet, J. (2011). Camera Calibration Toolbox for Matlab. Computational Vision at the California Institute of Technology.

Clavert, P., Kempf, J.F., Bonnomet, F., Boutemy, P., Marcelin, L. and Kahn, J.L. (2001). Effects of freezing/thawing on the biomechanical properties of human tendons. Surg Radiol Anat, 23(4):259 - 262.

Cobb, W., Kercher, K., and Heniford, B. (2005). The argument for lightweight polypropylene mesh in hernia repair. Surg Innov, 12:63 - 69.

Cooney, G., Moerman, K., Takaza, M., Winter, Des C., Simms and C. K. (2015). Uniaxial and biaxial mechanical properties of porcine linea alba. Journal of the Mechanical Behavior of Biomedical Materials, 41:68 - 82 .

Dabbas, N., Adams, K., Pearson, K., and Royle, G. (2011). Frequency of abdominal wall hernias: is classical teaching out of date? JRSM short reports, 2(1):5.

Dumanian, G. (2007). Grabb and Smith's Plastic Surgery, Sixth Edition, chapter Abdominal wall reconstruction, pages 670-675. Lippincott Williams and Wilkins.

Förstemann, T., Trzewik, J., Holste, J., Batke, B., Konerding, M. a., Wolloscheck, T., and Hartung, C. (2011). Forces and deformations of the abdominal wall - A mechanical and geometrical approach to the linea alba. Journal of Biomechanics, 44(4):600 - 6 .

Giannini, S., Buda, R., Di Caprio, F., and et al. (2008). Effects of freezing on the biomechanical and structural properties of human posterior tibial tendons. International Orthopaedics., $32(2): 145-151$.

Greca, F. H., de Paula, J. B., Biondo-Simões, M. L., da Costa, F. D., da Silva, A. P., Time, S., and Mansur, A. (2001). The influence of differing pore sizes on the biocompatibility of two polypropylene meshes in the repair of abdominal defects. Experimental study in dogs. Hernia, 5(2):59 - 64 .

Hernández, B., Peña, E., Pascual, G., Rodríguez, M., Calvo, B., Doblaré, M., and Bellón, J. (2011). Mechanical and histological characterization of the abdominal muscle. a previous step to modelling hernia surgery. Journal of the Mechanical Behavior of Biomedical Materials, $4(3): 392-404$.

Hernández-Gascón, B., Peña, E., Melero, H., Pascual, G., Doblaré, M., Ginebra, M., Bellón, J., and Calvo, B. (2011). Mechanical behavior of synthetic surgical meshes: Finite element 
simulation of the herniated abdominal wall. Acta Biomaterialia, 7(11):3905 - 3913.

Hernández-Gascón, B., Peña, E., Pascual, G., Rodríguez, M., Bellón, J., and Calvo, B. (2012). Long-term anisotropic mechanical response of surgical meshes used to repair abdominal wall defects. Journal of the Mechanical Behavior of Biomedical Materials, 5(1):257 - 271.

Hwang, W., Carvalho, J. C., Tarlovsky, I., and Boriek, A. M. (2005). Passive mechanics of canine internal abdominal muscles. Journal of Applied Physiology, 98(5):1829 - 1835.

Kingsnorth, A. and LeBlanc, K. (2003). Hernias: inguinal and incisional. The Lancet, 362:1561 $-1571$.

Kirilova, M., Stoytchev, S., Pashkouleva, D., and Kavardzhikov, V. (2011). Experimental study of the mechanical properties of human abdominal fascia. Medical engineering 8 physics, $33(1): 1-6$.

Klinge, U., Klosterhalfen, B., Birkenhauer, V., Junge, K., Conze, J., and Schumpelick, V. (2002). Impact of polymer pore size on the interfaces scar formation in a rat model. J Surg Res, 103:208 - 214 .

Klosterhalfen, B., Junge, K., and Klinge, U. (2002). The lightweight and large porous mesh concept for hernia repair. Expert Rev Med Devices, 2(1):103 - 117.

LeBlanc, K., Bellanger, D., Rhynes, K., Baker, D., and Stout, R. (2002). Tissue attachment strength of prosthetic meshes used in ventral and incisional hernia repair. Surgical Endoscopy And Other Interventional Techniques, 16(11):1542-1546.

Lyons, M., Winter, D., and Simms, C. (2014). Mechanical characterisation of porcine rectus sheath under uniaxial and biaxial tension. Journal of biomechanics, 47(8):1876 - 84.

Martins, P., Peña, E., Jorge, R. M. N., Santos, A., Santos, L., Mascarenhas, T., and Calvo, B. (2012). Mechanical characterization and constitutive modelling of the damage process in rectus sheath. Journal of the Mechanical Behavior of Biomedical Materials, 8:111 - 122 .

Paajanen, H. and Hemunen, H. (2004). Long-term pain and recurrence after repair of ventral incisional hernias by open mesh: clinical and mri study. Langenbecks Arch Surg, 389:366 370.

Peeters, E., van Barneveld, K. W. Y., Schreinemacher, M. H., De Hertogh, G., Ozog, Y., Bouvy, N., and Miserez, M. (2013). One-year outcome of biological and synthetic bioabsorbable meshes for augmentation of large abdominal wall defects in a rabbit model. The Journal of surgical research, 180(2):274 - 83.

Podwojewski, F., Otténio, M., Beillas, P., Guérin, G., Turquier, F., and Mitton, D. (2013). Mechanical response of animal abdominal walls in vitro: evaluation of the influence of a hernia defect and a repair with a mesh implanted intraperitoneally. Journal of biomechanics, 46(3):561 - 6 .

Podwojewski, F., Otténio, M., Beillas, P., Guérin, G., Turquier, F., and Mitton, D. (2014). Mechanical response of human abdominal walls ex vivo: Effect of an incisional hernia and a mesh repair. Journal of the Mechanical Behavior of Biomedical Materials, 38:126 - 33.

Simón-Allué, R., Pérez-López, P., Sotomayor, S., Peña, E., Pascual, G., Bellón, J., and Calvo, B. (2014). Short- and long-term biomechanical and morphological study of new suture types in abdominal wall closure. Journal of the Mechanical Behavior of Biomedical Materials, 37:1 -11 .

Song, C., Alijani, A., Frank, T., Hanna, G., and Cuschieri, A. (2006). Mechanical properties of the human abdominal wall measured in vivo during insufflation for laparoscopic surgery. Surg Endosc, 20(6):987 - 990. 
Szymczak, C., Lubowiecka, I., Tomaszewska, A., and Smietański, M. (2012). Investigation of abdomen surface deformation due to life excitation: implications for implant selection and orientation in laparoscopic ventral hernia repair. Clinical Biomechanics (Bristol, Avon), $27(2): 105-10$.

Tran, D., Mitton, D., Voirin, D., Turquier, F., and Beillas, P. (2014). Contribution of the skin, rectus abdominis and their sheaths to the structural response of the abdominal wall ex vivo. Journal of Biomechanics, 47(12):3056 - 63.

Zhang, A., Cheng, S., Lei, D., He, L., Luo, D., and Gao, D. (2002). An experimental study of the mechanical behavior of frozen arteries at low temperatures. CryoLetters, 23(6):389 - 96. 